\title{
The multidisciplinary approach in the diagnosis of idiopathic pulmonary fibrosis: a patient case-based review
}

\author{
Sara Tomassetti ${ }^{1}$, Sara Piciucchi ${ }^{2}$, Paola Tantalocco ${ }^{1}$, Alessandra Dubini $^{3}$ and \\ Venerino Poletti ${ }^{1}$
}

Affiliations: ${ }^{1}$ Dept of Diseases of the Thorax, GB Morgagni Hospital, Forli, Italy. ${ }^{2}$ Dept of Radiology, GB Morgagni Hospital, Forli, Italy. ${ }^{3}$ Dept of Pathology, GB Morgagni Hospital, Forli, Italy.

Correspondence: Sara Tomassetti, Dept of Diseases of the Thorax, Via C. Forlanini, Forli, FC 34-47121, Italy. E-mail: s.tomassettidgmail.com

ABSTRACT Idiopathic pulmonary fibrosis (IPF) is a specific form of chronic, progressively fibrosing interstitial pneumonia that is associated with a significantly worse prognosis than other forms of chronic interstitial pneumonia. An early and accurate diagnosis of IPF is important to enable the initiation of disease-specific therapies, which have the potential to reduce disease progression, and the avoidance of inappropriate and potentially harmful drugs. Establishing an accurate diagnosis of IPF can be challenging. Recent studies and international guidelines advocate the importance of a multidisciplinary team (MDT) in the initial diagnostic assessment of patients with suspected IPF. Typical MDT members include a pulmonologist, a radiologist and a pathologist, with further input from a thoracic surgeon, a rheumatologist, a specialist nurse and an occupational physician where appropriate. Multidisciplinary diagnosis is considered the gold standard because it can improve the accuracy of diagnosis of IPF, avoid unnecessary testing (e.g. lung biopsy), and optimise patient management. Here we highlight the strengths and limitations of the multidisciplinary approach to IPF diagnosis through MDT discussion of two patient cases.

@ERSpublications

The strengths and limitations of IPF management by a multidisciplinary team are highlighted in two patient cases http://ow.ly/H04Xe

\section{Introduction}

Idiopathic pulmonary fibrosis (IPF) is the most prevalent and lethal condition in the family of idiopathic interstitial pneumonias, a subgroup of interstitial lung diseases (ILDs) [1, 2]. IPF is characterised by progressive respiratory failure resulting from a relentless fibrosis leading to destruction of the lung architecture. The specific molecular and cellular mechanisms and causes of disease onset and progression are still unknown [3]. However, a growing body of evidence suggests that IPF is caused by an aberrant wound healing response to recurrent alveolar epithelial cell injury causing accelerated lung senescence and an increased fibrosis [4-6]. Cigarette smoking, exposure to organic and inorganic dust, and genetic factors have been shown to play a central role in disease pathogenesis [7]. However, to date, molecular pathways, genetic abnormalities and environmental interactions involved in the disease pathogenesis have been only partially clarified. A specific disease mechanism-based profile (genetic/biomolecular) has not been identified.

Received: Nov 242014 | Accepted after revision: Dec 162014

The case studies detailed in this article are not intended to be representative of the efficacy and safety profile of pirfenidone.

Conflict of interest: Disclosures can be found alongside the online version of this article at err.ersjournals.com

Provenance: Publication of this peer-reviewed article was supported by InterMune International AG, Muttenz, Switzerland (article sponsor, European Respiratory Review issue 135).

Copyright OERS 2015. ERR articles are open access and distributed under the terms of the Creative Commons Attribution Non-Commercial Licence 4.0. 
Therefore, the diagnosis still relies on the presence of a radiological and/or histopathological pattern of usual interstitial pneumonia (UIP) [3].

This diagnostic approach results in areas of uncertainty, particularly in cases where the histological UIP pattern is insufficient to differentiate IPF from other entities that can present similar features, such as chronic hypersensitivity pneumonia (CHP) $[8,9]$. Another possible approach to ILD classification and diagnosis is to consider the disease behaviour [10]. However, IPF natural history may vary among individual patients and may follow one of several clinical courses [11-13]. The majority of patients have slowly progressive disease, some have an accelerated decline followed by death, and some have periods of relatively stable disease interposed with episodes of acute respiratory worsening. In this complex scenario, IPF diagnosis may be challenging. Here we present two clinical cases. The first is a clinical case of a straightforward diagnosis of IPF, but with a slow progression and good long-term prognosis after early initiation and long-term treatment with pirfenidone. The second clinical case exemplifies a very challenging differential diagnosis between IPF and CHP in which neither the multidisciplinary team (MDT) of experts, nor the disease behaviour approach, leave us with the final impression of having framed this complex clinical picture in a successful and timely manner.

\section{Diagnostic criteria}

According to international guidelines, the diagnosis of IPF requires the exclusion of other known causes of ILD and the presence of a UIP pattern on high-resolution computed tomography (HRCT), or specific combinations of HRCT and surgical lung biopsy (SLB) patterns in patients lacking typical HRCT features [3]. Current guidelines acknowledge the areas of uncertainty in IPF diagnosis and for patients who do not meet the criteria for a definite UIP pattern, there are two other classifications: a possible UIP pattern or inconsistent with UIP pattern [3].

HRCT scanning can provide a confident, highly specific diagnosis of IPF in $\sim 50-60 \%$ of patients with diffuse lung disease [14-16]. In the remaining $40-50 \%$ of patients with suspected IPF, HRCT findings do not reveal a typical UIP pattern. In this scenario, guidelines recommend proceeding directly to SLB. However, this procedure is associated with significant risks and there is a growing body of studies showing that less invasive diagnostic methods, particularly transbronchial lung cryobiopsy (TBLC), may be used to diagnose UIP [17-19].

The histological lesion of IPF is that of UIP. Typical findings are marked fibrosis with architectural distortion, with or without honeycombing in a predominantly subpleural and paraseptal distribution, and patchy involvement of the lung parenchyma by fibroblast foci [20]. Since the UIP pattern is not specific for IPF, the ultimate diagnosis of IPF will rely on the interpretation of specific combinations of clinical, radiological, pathological and laboratory data by the MDT $[3,16]$.

\section{The challenge of diagnosis}

The diagnosis of IPF, as outlined above, requires information from several sources to be interpreted and integrated, and this can pose significant challenges [21]. For example, inadequacies in the initial patient examination and history may cause the physician to overlook alternative diagnoses and exposures to other causes of ILD. Certain diagnostic features, such as honeycombing on HRCT, are subjective and show significant interobserver variability [22]. Indeed, across the range of ILDs, histological findings lack the diagnostic sensitivity, specificity and reproducibility to be sufficiently pathognomonic in isolation [3]. Furthermore, in some cases IPF and other chronic ILDs, particularly CHP, can be indistinguishable. It has been recently outlined that a thorough investigation of occult putative environmental causes may reveal an unrecognised cause, most frequently avian antigen exposure [9]. As a consequence a large proportion of IPF cases may be reclassified as CHP. However, it remains unclear whether this meticulous but debatable approach, aiming to reclassify occult CHP, leads to a different management approach associated with an additional benefit for patients. To understand this we need to further investigate the differences between UIP patterns in different clinical entities, uncovering specific genetic and molecular mechanisms that drive the natural history of different fibrotic ILDs and potentially provide novel targets for anti-fibrotic drugs. In the future, a pathogenetic approach may allow us to avoid the limitations of both the aetiology and MDT-based diagnostic approaches. This is undoubtedly the future of IPF, but currently our knowledge of the molecular phenotypes of IPF patients is insufficient to enable this mechanistic approach. The current MDT approach is the best possible surrogate of an accurate diagnosis of IPF, particularly for cases of challenging differential diagnosis.

\section{The role of the MDT}

During the past decade, there has been increasing appreciation that the diagnosis of IPF is best achieved by a MDT that comprises clinicians, thoracic radiologists and thoracic pathologists [22]. This is reflected 
in the 2011 international guidelines on the management of IPF, which state: "Careful exclusion of alternative aetiologies through multidisciplinary discussion between pulmonologists, radiologists, and pathologists experienced in the diagnosis of ILD is of the utmost importance to an accurate diagnosis" [3]. The guidelines add that multidisciplinary discussion is particularly relevant in cases in which the radiological and histopathological patterns are discordant. According to the recommended approach to the diagnostic assessment of patients with suspected IPF (fig. 1), initial assessment is targeted at confirming a diagnosis while subsequent investigations aim to quantify disease severity and to exclude disease-specific complications or other organ involvement.

There is a small but growing evidence base to support the value of the MDT approach to managing patients with IPF. FLAHERTY et al. [22] showed that a consensus diagnosis, reached after a careful exchange of clinical, radiographic and histopathological information, often differed from the initial diagnosis reached by the individual team members working in isolation. They concluded that "dynamic interactions between clinicians, radiologists, and pathologists improve interobserver agreement and diagnostic confidence" [22].

In a separate study, the group showed that there is significant disagreement in the diagnosis of IPF between physicians based in local centres and those in referral centres. In both settings, the introduction of an interactive MDT approach improved diagnostic agreement [23]. A large European study found that the overall accuracy of diagnosis of IPF in expert centres is good, at 87.2\% [24]. However, the level of agreement within the panels was only fair-to-moderate, highlighting the importance of including MDT members with specific expertise and familiarity with ILD [22]. There is also evidence to suggest that specialist centres running "ILD MDTs" can improve clinical outcomes, presumably through better case identification and earlier or more appropriate treatment. In a study by LAMAs et al. [25] delayed access to a specialist centre was associated with higher mortality in IPF, independently of disease severity.

Here we describe two patient cases and illustrate the value of multidisciplinary interpretation of clinical, radiological and histological findings for a more confident diagnosis of ILDs.

\section{Patient case 1 \\ Clinical details}

A 63-year-old man, working as a train driver, presented with a 3-year history of chronic dry cough and with bilateral crackles. He was a lifelong nonsmoker and had an unremarkable family history. His past medical history included a left pleural effusion developed at 2 years of age and a positive tuberculosis skin-test result. He presented with no comorbidities and was under no medication. Pulmonary function tests (PFTs) were within the normal limits except for a mild reduction in diffusing capacity of the lung for carbon monoxide (DLCO) (69\% predicted). No oxygen desaturation was revealed during 6-min walk tests (6MWTs); resting oxygen saturation was $97 \%$ and 6-min walking distance (6MWD) was $420 \mathrm{~m}$. Laboratory tests were normal and autoimmunity and precipitin screens were negative.

\section{HRCT findings}

A HRCT scan was performed to investigate suspected IPF. The images (fig. 2) showed subpleural mild reticulation, possible bronchiectasis and no air trapping. Coarse reticulation together with the absence of

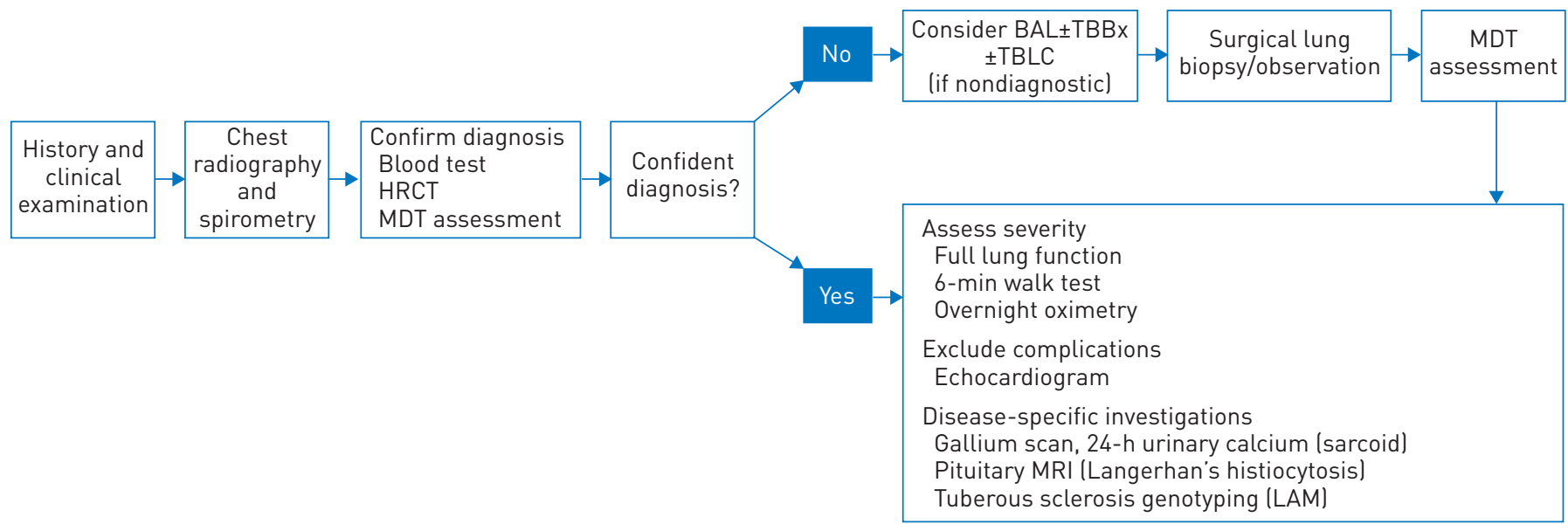

FIGURE 1 Investigation of suspected idiopathic pulmonary fibrosis: multidisciplinary team (MDT) discussion is a key component of the diagnostic pathway. HRCT: high-resolution computed tomography; BAL: bronchoalveolar lavage; TBBx: transbronchial biopsy; TBLC: transbronchial lung cryobiopsy; MRI: magnetic resonance imaging; LAM: lymphangioleiomyomatosis. Reproduced and modified from [16] with permission from the publisher. 

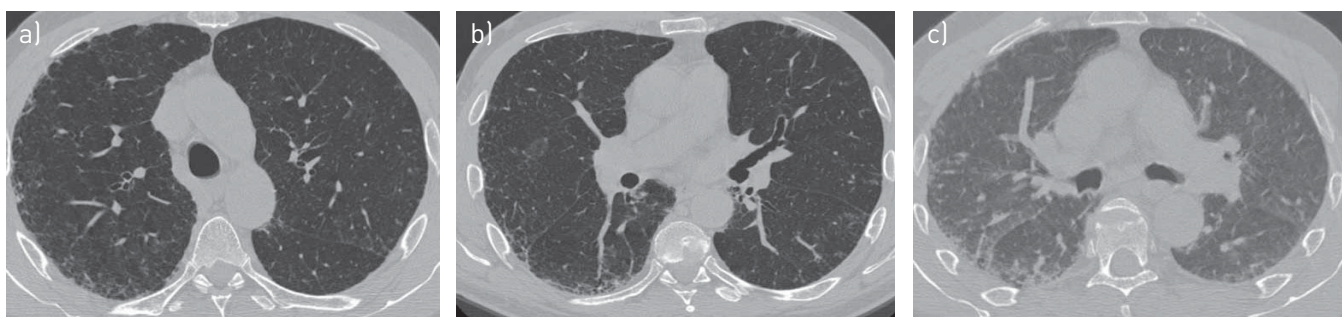

FIGURE $2 \mathrm{a}-\mathrm{c}$ ) Transverse high-resolution computed tomography images showing a possible usual interstitial pneumonia pattern in patient case 1.

cystic spaces and honeycombing suggested a possible UIP pattern, according to the American Thoracic Society (ATS)/European Respiratory Society (ERS)/Japanese Respiratory Society (JRS)/Latin American Thoracic Association (ALAT) criteria (table 1).

\section{Surgical lung biopsy}

Based on the HRCT findings suggesting a possible UIP pattern, a SLB of the right upper and lower lobes was performed. Pathological analysis of biopsy samples (fig. 3) revealed patchy fibrosis, honeycombing, fibroblastic foci and areas of pulmonary ossification. The patient fulfilled all the histopathological criteria for UIP pattern, according to the ATS/ERS/JRS/ALAT criteria (table 2) [3].

\section{Diagnosis and treatment}

After discussion by the MDT, the patient was diagnosed with IPF with a high degree of confidence. He was offered two options: triple therapy with azathioprine, prednisolone and $N$-acetylcysteine (NAC) or enrolment in the CAPACITY (Clinical Studies Assessing Pirfenidone in IPF: Research of Efficacy and Safety Outcomes) trial. At that time, the safety concerns about triple therapy were still unknown (the triple therapy arm of the PANTHER-IPF (Prednisone, Azathioprine, $N$-acetylcysteine: a Study That Evaluates Response in IPF) trial was discontinued early in October 2011 due to increased rates of death and hospitalisation) [26]. The patient decided to participate in the CAPACITY trial and was randomised to receive treatment with pirfenidone or placebo.

\section{Long-term follow-up}

According to PFTs and clinical examination, his disease remained relatively stable for 6 years on pirfenidone treatment but mild progression was noted in the last year of treatment with worsening DLCO, forced vital capacity (FVC) and 6MWT. Oxygen saturation during the last 6MWT was 85\%. HRCT follow-up was performed at both 3 and 7 years after the start of pirfenidone treatment. After 3 years, HRCT scans (fig. $4 \mathrm{a}-\mathrm{c}$ ) revealed slight progression of the disease and showed moderate honeycombing in the right upper lobe, an increase in traction bronchiectasis and the development of honeycombing in the lower lobes. After 7 years, HRCT (fig. 4d and e) revealed further progression of the disease characterised by marked honeycombing in the right upper lobes and in both the left and right lower lobes.

TABLE 1 High-resolution computed tomography criteria for UIP pattern as specified in the 2011 ATS/ERS/JRS/ALAT guidelines for the diagnosis of interstitial lung disease

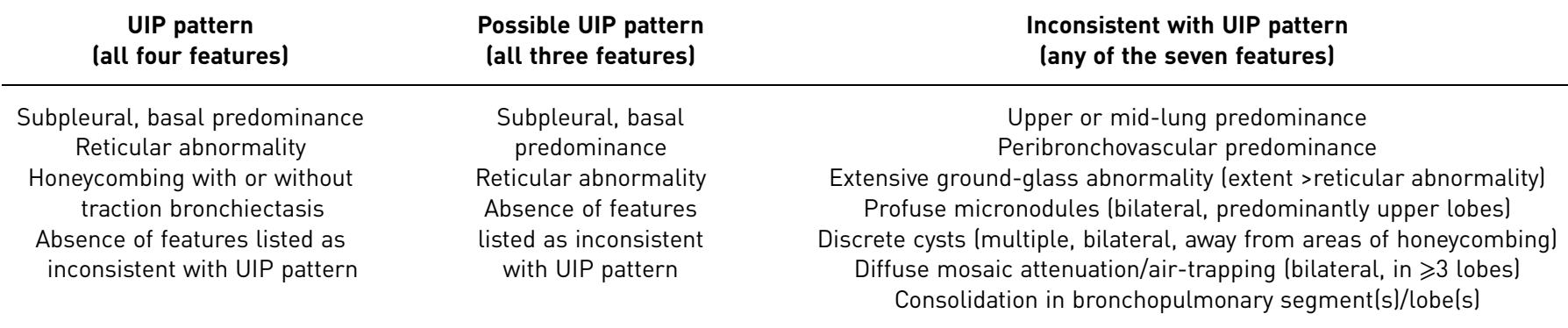

\section{Inconsistent with UIP pattern (any of the seven features)}

UIP: usual interstitial pneumonia; ATS: American Thoracic Society; ERS: European Respiratory Society; JRS: Japanese Respiratory Society; ALAT: Latin American Thoracic Association. Reproduced and modified from [3] with permission from the publisher. 

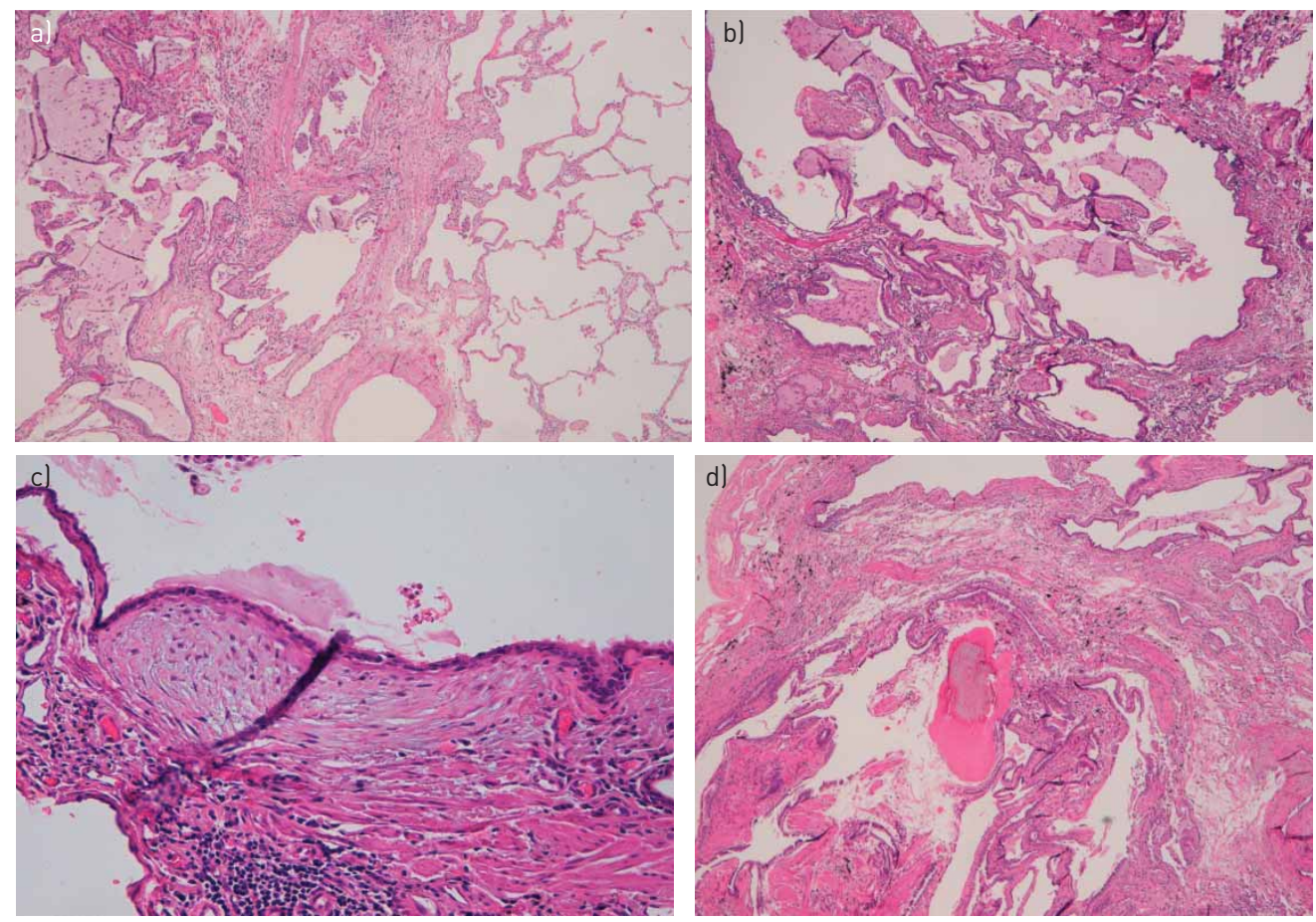

FIGURE 3 Microscopy of the surgical lung biopsy for patient case 1 showing a) patchy fibrosis, b) honeycombing c) fibroblastic foci, and d) areas of pulmonary ossification.

TABLE 2 Combination of HRCT and surgical lung biopsy for the diagnosis of IPF (requires MDT discussion]

\begin{tabular}{|c|c|c|}
\hline HRCT pattern & Surgical lung biopsy pattern" (when performed) & Diagnosis of IPF? \\
\hline \multirow[t]{5}{*}{ UIP } & UIP & Yes \\
\hline & Probable UIP & Yes \\
\hline & Possible UIP & Yes \\
\hline & Nonclassifiable fibrosis $^{+}$ & Yes \\
\hline & Not UIP & No \\
\hline \multirow[t]{4}{*}{ Possible UIP } & UIP & Yes \\
\hline & Probable UIP & Yes \\
\hline & Possible UIP & Probable $e^{\S}$ \\
\hline & Nonclassifiable fibrosis & Probable ${ }^{\S}$ \\
\hline \multirow[t]{5}{*}{ Inconsistent with UIP } & UIP & Possible $\S^{\S}$ \\
\hline & Probable UIP & No \\
\hline & Possible UIP & No \\
\hline & Nonclassifiable fibrosis & No \\
\hline & Not UIP & No \\
\hline
\end{tabular}

Certain combinations of high-resolution computed tomography (HRCT) and surgical lung biopsy patterns correspond with a diagnosis of idiopathic pulmonary fibrosis (IPF) (a yes in the far right column). For example, the combination of usual interstitial pneumonia (UIP) HRCT and probable UIP or possible UIP or nonclassifiable fibrosis patterns on surgical lung biopsy equals a diagnosis of IPF; the combination of UIP HRCT and not UIP surgical lung biopsy pattern does not make the diagnosis of IPF. \#: patterns are as described in table 1. ": the accuracy of the diagnosis of IPF increases with multidisciplinary team (MDT) discussion. This is particularly relevant in cases in which the radiological and histopathological patterns are discordant (e.g. HRCT is inconsistent with UIP and histopathology is UIP). There are data to suggest that the accuracy of diagnosis is improved with MDT discussion among interstitial lung disease (ILD) experts compared with clinician specialists in the community setting [13]; timely referral to ILD experts is encouraged. ${ }^{+}$: some biopsies may reveal a pattern of fibrosis that does not meet the criteria for UIP pattern and the other idiopathic interstitial pneumonias [19]. These biopsies may be termed "nonclassifiable fibrosis." §: MDT discussion should include discussions of the potential for sampling error and a re-evaluation of adequacy of the HRCT technique. In cases with an "inconsistent with UIP" HRCT pattern and a "UIP" surgical lung biopsy pattern, the possibility of a diagnosis of IPF still exists and clarification by MDT discussion among ILD experts is indicated. Reproduced and modified from [3] with permission from the publisher. 

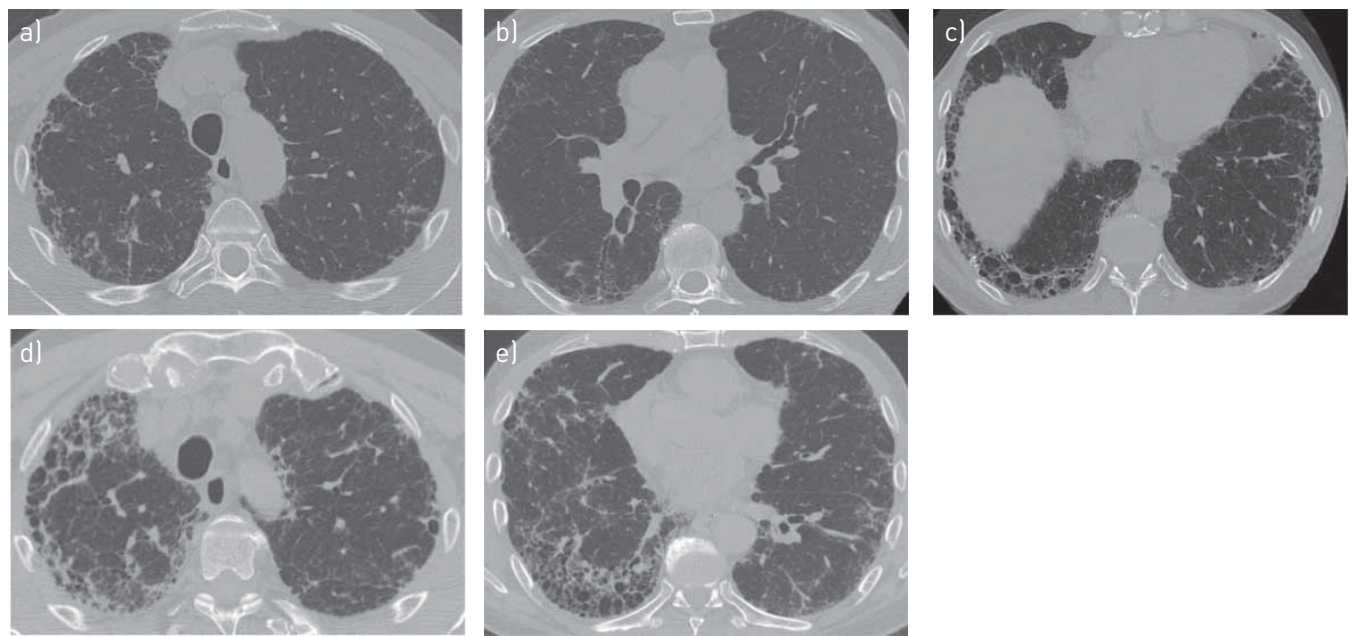

FIGURE 4 High-resolution computed tomography follow-up of patient case 1 at a-c) 3 years, and d, e) 7 years after the start of treatment showing disease progression.

Pirfenidone was generally well tolerated by the patient, with no significant adverse events occurring during the 7 years on treatment. The patient is now 70 years old and enjoys a good quality of life. He does not require oxygen and can continue everyday activities without notable restrictions.

\section{Patient case 2}

Clinical details

A 64-year-old man, working as a builder, presented with an 8-month history of chronic dry cough and dyspnoea on exertion (modified Medical Research Council dyspnoea scale=2). Chest auscultation revealed bibasilar crackles and no clubbing or peripheral oedema were evident. He was an ex-smoker (12 pack-years) who had stopped smoking 5 years earlier and had an unremarkable family history. His only comorbidity was diabetes, which was controlled through dietary management and was under no medication. The patient did not report any symptoms of gastro-oesophageal reflux disease (GORD). After a careful review of his exposure history, the patient admitted to feeding pigeons occasionally when they flew into his garden but denied breeding them in his house. Blood tests were normal, except for a mild elevation in lactate dehydrogenase, positive anti-nuclear autoantibodies and positive precipitins for pigeon droppings. PFTs revealed a moderate reduction in DLCO (43\% predicted) and no oxygen desaturation on 6MWT (6MWD was $420 \mathrm{~m}$ ).

\section{HRCT findings}

HRCT at presentation (fig. 5) showed coarse reticulation and areas of honeycombing in the right upper lobe as well as mild reticulation in the periphery of the left lung. HRCT scans revealed asymmetrical disease, based on the lower volume of the right lobe compared with the other hemithorax. The lower lobe (particularly the right one) showed severe fibrosis, with coarse reticulation and questionable honeycombing, with mosaic attenuation. Asymmetrical disease was also confirmed in the costophrenic angles with mild reticulation on one side and fibrosis on the other. Expiratory images revealed air trapping in areas where mosaic attenuation had been identified. On the basis of an absent apico-basal gradient
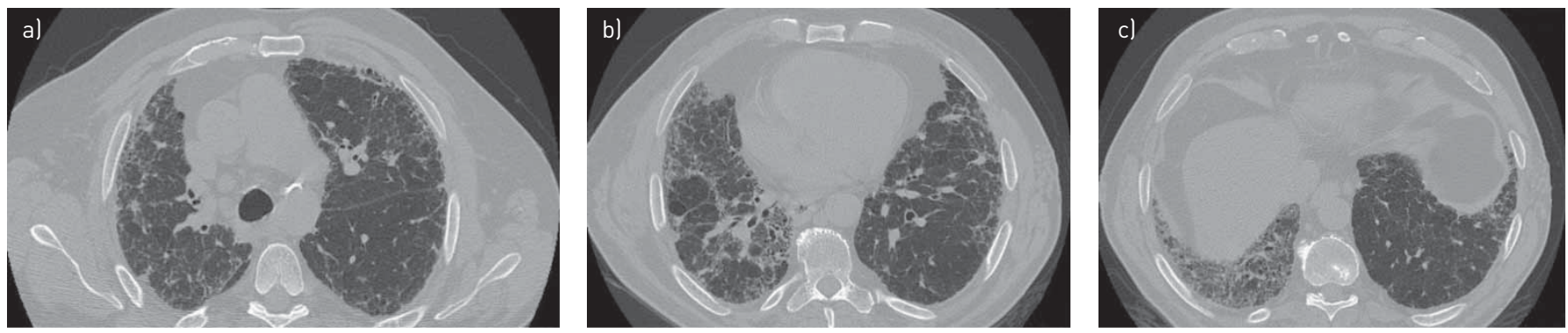

FIGURE $5 \mathrm{a}-\mathrm{c}$ ) Transverse high-resolution computed tomography images showing a possible usual interstitial pneumonia pattern in patient case 2. 

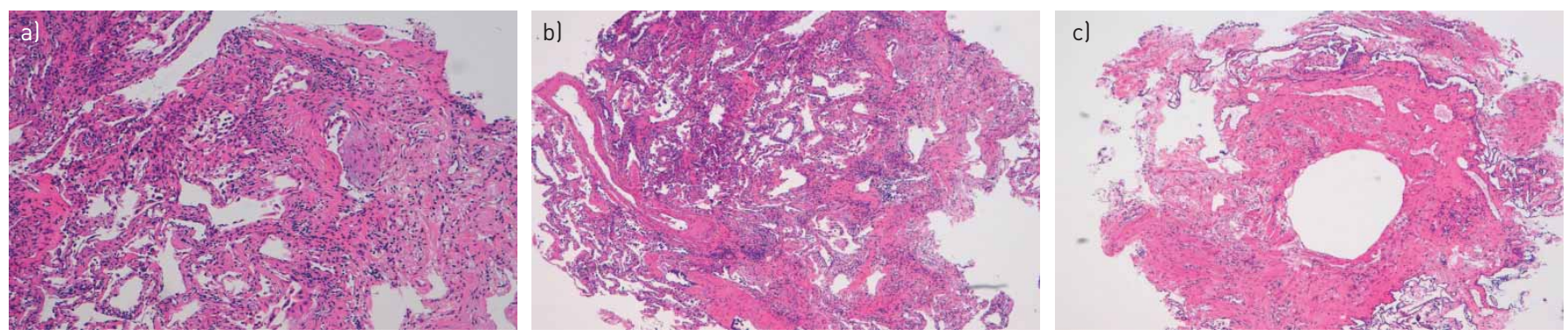

FIGURE 6 Microscopy of transbronchial lung cryobiopsy for patient case 2 showing a, b) fibroblastic foci and patchy fibrosis, and c) subpleural dense scarring.

and the mosaic attenuation HRCT findings, these were considered to be inconsistent with a UIP pattern (table 1) and suggestive of possible CHP. Additional ancillary HRCT findings included moderate enlargement of the pulmonary artery and hiatal hernia.

\section{Bronchoalveolar lavage}

Bronchoalveolar lavage (BAL) was performed and showed no lymphocytosis. Oil red-positive macrophages were consistently reported. Together with the hiatal hernia and the asymmetric fibrosis, BAL findings suggested possible GORD, even in the absence of symptoms.

\section{Transbronchial lung cryobiopsy}

The MDT found it very challenging to differentiate between IPF and CHP, and therefore decided to perform a TBLC. Pathological analysis of biopsy samples (fig. 6) showed the presence of fibroblastic foci, patchy fibrosis and subpleural dense scarring, i.e. a characteristic UIP pattern without any ancilliary features suggestive of CHP.

\section{MDT discussion and treatment}

After discussion by the MDT, however, the combination of clinical HRCT and pathological findings was found to be insufficient to allow a confident diagnosis, due to not meeting the ATS/ERS/JRS/ALAT criteria for a definite diagnosis of IPF [3].

The MDT decided also to assess the comorbidities in more detail. An endoscopy was performed which confirmed severe GORD and oesophagitis. Echocardiography did not show significant pulmonary hypertension. Anti-reflux therapy was started. Based on an uncertain IPF diagnosis, triple therapy with steroids, NAC and azathioprine was initiated. Safety concerns about triple therapy were still unknown at that time (PANTHER-IPF trial, 2011) [26]. During the following 12 months the patient experienced an episode of acute bronchitis and a further decline both in FVC and in DLCO.

\section{Follow-up HRCT}

1 year after the start of treatment with triple therapy, HRCT scans (fig. 7) showed a UIP pattern with asymmetric disease and the diagnosis of IPF was confirmed by the MDT. At that point, the disease was already severe and the patient was switched to treatment with pirfenidone. Thereafter, he experienced an acute exacerbation, which was believed to be triggered by his chronic inhalation problems, and was finally transplanted.
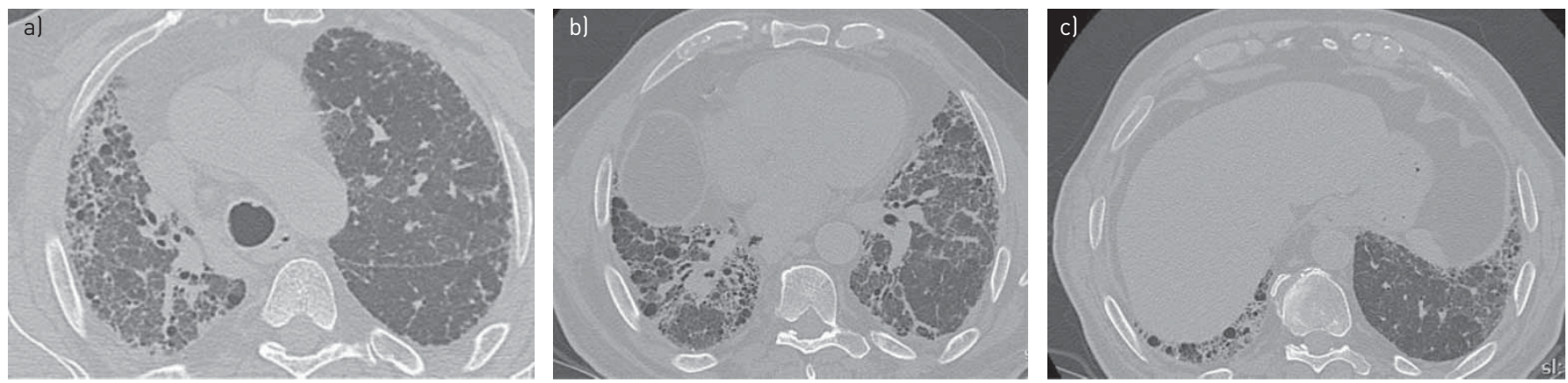

FIGURE 7 a-c) High-resolution computed tomography follow-up of patient case 2 at 1 year after the start of treatment showing a definite usual interstitial pneumonia pattern. 


\section{Discussion}

Here we report two clinical cases which highlight the value of MDT discussion in the diagnosis and management of IPF. The first case illustrates the utility of the 2011 ATS/ERS/JRS/ALAT criteria and the recommendation for MDT discussion in enabling an early diagnosis of definite IPF and early enrolment into the CAPACITY study. This was particularly important in this case since it was not possible to diagnose IPF purely based on HRCT, which showed a possible UIP pattern. This necessitated SLB, in line with current guidelines, and MDT discussion of clinical, radiological and histological features to reach a definitive diagnosis. This case underscores findings in the literature indicating that characteristic HRCT features are not present in $\sim 30-40 \%$ of IPF patients and that lung biopsy may be needed to confirm diagnosis [27]. There is a growing body of evidence suggesting that subjects with probable IPF, accounting for $\sim 16 \%$ of cases, are likely to have UIP pattern on lung biopsy $(82 \%)[28,29]$. Interestingly, FeLL et al. [30] showed that in patients aged over 65 years with reticular abnormalities but no honeycombing, as described in the case above, the diagnostic likelihood of IPF exceeded 95\%.

The second case highlights certain limitations with the application of guideline recommendations. The guidelines focus primarily on "definite" IPF, which is most often diagnosed from typical HRCT appearances. The definition of "probable" and "possible" IPF is an advance, but there is a lack of management guidance for these highly prevalent clinical scenarios. Patients with "probable" or "possible" IPF are frequently encountered in clinical practice and often have a differential diagnosis of fibrotic nonspecific interstitial pneumonia or CHP [8], and less frequently with other rare entities [8, 31]. However, the guidelines do recognise that when the radiological and histopathological patterns are discordant (e.g., HRCT is inconsistent with UIP and histopathology is UIP), the possibility of a diagnosis of IPF still exists and clarification by MDT discussion among ILD experts is indicated [3]. Serological tests may be appropriate in this scenario, although it should be noted that serum precipitins have a controversial diagnostic value due to their limited sensitivity, specificity, and positive and negative predictive values $(76 \%, 82 \%, 69.5 \%$ and $86.4 \%$, respectively) [32].

Biopsy can be helpful in differentiating IPF from CHP, but with some limitations. While there are histological findings that may suggest the presence of CHP, none of these features are specific as they can also be found (albeit less frequently) in IPF. A minority of CHP (8\%) are pathologically indistinguishable from IPF, because they present with identical UIP features. It is still unclear what the different pathological patterns found in cases of CHP mean in terms of prognosis, and whether CHP with a UIP-like pattern behaves differently from idiopathic UIP [33]. In this case, MDT discussion and follow-up HRCT did eventually enable a more confident diagnosis of IPF [34]. However, at this stage, the potential benefit of pirfenidone may have been limited by the ongoing progression and severity of disease.

The correct diagnosis of IPF has become particularly important in view of the availability of effective IPF-specific therapies and the demonstration that a previously widely used immunosuppressive treatment regimen in IPF (prednisone, azathioprine and NAC) increases the risks of hospitalisation and death [26]. Thus, an early and accurate diagnosis of IPF through MDT discussion is essential to enable the initiation of therapies that have the potential to reduce disease progression and the avoidance of inappropriate and potentially harmful drugs.

\section{Acknowledgements}

This article is based on the proceedings of a satellite symposium held at the 2014 ERS International Congress (Munich, Germany), which was sponsored by InterMune Inc. The authors were assisted in the preparation of the text by professional medical writers at IntraMed International (Milan, Italy). The medical writing support was funded by InterMune International AG (Muttenz, Switzerland).

\section{References}

1 Nalysnyk L, Cid-Ruzafa J, Rotella P, et al. Incidence and prevalence of idiopathic pulmonary fibrosis: review of the literature. Eur Respir Rev 2012; 21: 355-361.

2 Travis WD, Costabel U, Hansell DM, et al. An official American Thoracic Society/European Respiratory Society statement: update of the international multidisciplinary classification of the idiopathic interstitial pneumonias. Am J Respir Crit Care Med 2013; 188: 733-748.

3 Raghu G, Collard HR, Egan JJ, et al. An official ATS/ERS/JRS/ALAT statement: idiopathic pulmonary fibrosis: evidence-based guidelines for diagnosis and management. Am J Respir Crit Care Med 2011; 183: 788-824.

4 Selman M, King TE, Pardo A, et al. Idiopathic pulmonary fibrosis: prevailing and evolving hypotheses about its pathogenesis and implications for therapy. Ann Intern Med 2001; 134: 136-151.

5 Chilosi M, Carloni A, Rossi A, et al. Premature lung aging and cellular senescence in the pathogenesis of idiopathic pulmonary fibrosis and COPD/emphysema. Transl Res 2013; 162: 156-173.

6 Tomassetti S, Gurioli C, Ryu JH, et al. The impact of lung cancer on survival of idiopathic pulmonary fibrosis. Chest 2015; 147: 157-164.

7 Spagnolo P, Rossi G, Cavazza A. Pathogenesis of idiopathic pulmonary fibrosis and its clinical implications. Expert Rev Clin Immunol 2014; 10: 1005-1017. 
8 Sverzellati N, Wells AU, Tomassetti S, et al. Biopsy-proved idiopathic pulmonary fibrosis: spectrum of nondiagnostic thin-section CT diagnoses. Radiology 2010; 254: 957-964.

9 Morell F, Villar A, Montero MÁ, et al. Chronic hypersensitivity pneumonitis in patients diagnosed with idiopathic pulmonary fibrosis: a prospective case-cohort study. Lancet Respir Med 2013; 1: 685-694.

10 Ryerson CJ, Collard HR. Update on the diagnosis and classification of ILD. Curr Opin Pulm Med 2013; 19: 453-459.

11 Ley B, Collard HR, King TE Jr. Clinical course and prediction of survival in idiopathic pulmonary fibrosis. Am J Respir Crit Care Med 2011; 183: 431-440.

12 Poletti V, Ravaglia C, Buccioli M, et al. Idiopathic pulmonary fibrosis: diagnosis and prognostic evaluation. Respiration 2013; 86: 5-12.

13 Ryu JH, Moua T, Daniels CE, et al. Idiopathic pulmonary fibrosis: evolving concepts. Mayo Clin Proc 2014; 89: $1130-1142$.

14 Gotway MB, Freemer MM, King TE Jr. Challenges in pulmonary fibrosis. 1: use of high resolution CT scanning of the lung for the evaluation of patients with idiopathic interstitial pneumonias. Thorax 2007; 62: 546-553.

15 Raghu G, Lynch D, Godwin JD, et al. Diagnosis of idiopathic pulmonary fibrosis with high-resolution CT in patients with little or no radiological evidence of honeycombing: secondary analysis of a randomised, controlled trial. Lancet Respir Med 2014; 2: 277-284.

16 Maher TM. A clinical approach to diffuse parenchymal lung disease. Immunol Allergy Clin North Am 2012; 32: 453-472.

17 Casoni GL, Tomassetti S, Cavazza A, et al. Transbronchial lung cryobiopsy in the diagnosis of fibrotic interstitial lung diseases. PLoS One 2014; 9: e86716.

18 Tomassetti S, Cavazza A, Colby TV, et al. Transbronchial biopsy is useful in predicting UIP pattern. Respir Res 2012; 13: 96.

19 Poletti V, Casoni GL, Gurioli C, et al. Lung cryobiopsies: a paradigm shift in diagnostic bronchoscopy? Respirology 2014; 19: 645-654.

20 Valeyre D. Towards a better diagnosis of idiopathic pulmonary fibrosis. Eur Respir Rev 2011; 20: 108-113.

21 Wells AU. Managing diagnostic procedures in idiopathic pulmonary fibrosis. Eur Respir Rev 2013; 22: 158-162.

22 Flaherty KR, King TE Jr, Raghu G, et al. Idiopathic interstitial pneumonia: what is the effect of a multidisciplinary approach to diagnosis? Am J Respir Crit Care Med 2004; 170: 904-910.

23 Flaherty KR, Andrei AC, King TE Jr, et al. Idiopathic interstitial pneumonia: do community and academic physicians agree on diagnosis? Am J Respir Crit Care Med 2007; 175: 1054-1060.

24 Thomeer M, Demedts M, Behr J, et al. Multidisciplinary interobserver agreement in the diagnosis of idiopathic pulmonary fibrosis. Eur Respir J 2008; 31: 585-591.

25 Lamas DJ, Kawut SM, Bagiella E, et al. Delayed access and survival in idiopathic pulmonary fibrosis: a cohort study. Am J Respir Crit Care Med 2011; 184: 842-847.

26 Raghu G, Anstrom KJ, King TE Jr, et al. Prednisone, azathioprine, and $\mathrm{N}$-acetylcysteine for pulmonary fibrosis. N Engl J Med 2012; 366: 1968-1977.

27 Kaarteenaho R. The current position of surgical lung biopsy in the diagnosis of idiopathic pulmonary fibrosis. Respir Res 2013; 14: 43.

28 Chung JH, Chawla A, Peljto AL, et al. CT findings of probable UIP have a high predictive value for histologic UIP. Chest 2015; 147: 450-459.

29 Wells AU. The revised ATS/ERS/JRS/ALAT diagnostic criteria for idiopathic pulmonary fibrosis (IPF) - practical implications. Respir Res 2013; 14: Suppl. 1, S2.

30 Fell CD, Martinez FJ, Liu LX, et al. Clinical predictors of a diagnosis of idiopathic pulmonary fibrosis. Am J Respir Crit Care Med 2010; 181: 832-837.

31 Piciucchi S, Tomassetti S, Casoni G, et al. High resolution CT and histological findings in idiopathic pleuroparenchymal fibroelastosis: features and differential diagnosis. Respir Res 2011; 12: 111.

32 Fenoglio CM, Reboux G, Sudre B, et al. Diagnostic value of serum precipitins to mould antigens in active hypersensitivity pneumonitis. Eur Respir J 2007; 29: 706-712.

33 Churg A, Sin DD, Everett D, et al. Pathologic patterns and survival in chronic hypersensitivity pneumonitis. Am Surg Pathol 2009; 33: 1765-1770.

34 Wuyts WA, Cavazza A, Rossi G, et al. Differential diagnosis of usual interstitial pneumonia: when is it truly idiopathic? Eur Respir Rev 2014; 23: 308-319. 